\title{
The evaluation of the thermal storage electric heating load adjustability
}

\author{
Lu Jing $^{1, *}$, Li Suxiu ${ }^{1}$ and Kong Weizheng ${ }^{1}$ \\ ${ }^{1}$ State Grid Energy Research Institute Co. LTD, Beijing, China
}

\begin{abstract}
In recent years, in order to solve the environmental pollution problem caused by coal-fired heating in the heating area of north of china, electric heating has received extensive attention and has gradually been developed vigorously. Considering the building characteristics, outdoor temperature, indoor comfort and other factors, the building heat load adjustability model is established, and the heat storage device's load adjustability model considering the current operating status and heat storage capacity of the heat storage device is established, and the two are added together An evaluation model of the adjustability of regenerative electric heating users' load is obtained. This model not only considers the user comfort and the adjustment potential of the building where it is located, but also considers the adjustment potential of the heat storage device, which can more comprehensively and accurately evaluate the user load adjustment potential of thermal storage electric heating.
\end{abstract}

\section{Introduction}

In recent years, in order to solve the environmental pollution problem caused by coal-fired heating in the heating area of north of china, electric heating has received extensive attention and has gradually been developed vigorously ${ }^{[1]}$. Regenerative electric heating systems, on the one hand, directly provide thermal energy to independent heating pipe networks through electric boilers; on the other hand, they are equipped with certain heat storage devices to store thermal energy during low load periods and release heat energy during peak load periods. For electric heating facilities such as air conditioners and heat pumps, there have been certain studies on the evaluation of the load adjustability of residential electric heating users. Regarding electric heating equipment such as thermal storage electric boilers, due to its own thermal storage device, it can provide a certain load adjustment capability, and most of them only consider the adjustability of the thermal storage device itself, without considering the building adjustment potential. Regenerative electric heating system as a typical adjustable load, its adjustability is mainly manifested in two aspects, one is the adjustability of the heat storage device, and the other is the adjustability of the building's thermal load. This paper considers the building characteristics, outdoor temperature, indoor comfort and other factors to establish the building thermal load adjustability model, considers the current operating state of the thermal storage device and the thermal storage capacity to establish the thermal storage device's load adjustability, and the two are added together to obtain the thermal storage type Electric heating user load adjustability evaluation model.

\section{Heat load model}

The building heat load model mainly includes three methods, the unit area load index method, the statistical model method and the physical simulation method. The unit area load index method refers to multiplying the building unit area load index by the corresponding building area ${ }^{[2]}$. This method is simple and convenient, and is a commonly used method in engineering. However, the method obtains a static thermal load and cannot reflect the time dynamic characteristics of the thermal load. The statistical model is based on historical data and uses statistical analysis and other data processing techniques to obtain the relationship between the pre-influencing factors of the building's thermal load, and then obtain the thermal load mode ${ }^{[3]} 1$. This method mainly includes regression analysis, time series, artificial neural network, and Support vector machine method and gray theory, etc., this method requires a large amount of historical data as accumulation. The physical simulation method is to simulate the composition of the building and the heating system and the complex thermal dynamic interaction process between the subsystems according to the meteorological parameters and building characteristics ${ }^{[4]}$. The physical model can capture enough details to simulate the energy use of the electric heating strategy for the entire building. However, this modeling method requires complex parameters, high sensitivity, and excessive calculations. A simplified physical simulation method is used to model the heat load in this paper.

*Corresponding author: lujing_xjtu@163.com 
The time-varying equation of air temperature in the building space formed by the ordinary envelope structure is:

$$
\begin{gathered}
C_{\mathrm{a}} \frac{\mathrm{d} T_{\mathrm{in}}}{\mathrm{d} t}=\sum_{j=1}^{n} q_{\mathrm{wall}, j(t)}+q_{\mathrm{win}(t)}+q_{\mathrm{cov}(t)}+q_{\mathrm{vent}(t)}+q_{\mathrm{have}(t)} \\
t=1,2, \cdots, 24
\end{gathered}
$$

Where $C_{\mathrm{a}}$ is the heat capacity of the air in the building space; $q_{\text {wall, } j(t)}$ is the heat transferred through the heat of the first wall at the moment $t, n$ is the total number of walls; $q_{\text {win }(t)}$ is the heat radiated by the windows at the moment $\mathrm{t} ; q_{\operatorname{cov}(t)}$ is the heat transferred to the air by convection from the indoor heat source at the moment $\mathrm{t} ; q_{\mathrm{vent}(t)}$ is the heat brought into the room is exchanged between indoor and outdoor air or adjacent room air at all times; $q_{\text {have }(t)}$ is the heat sent into the room by the heating system at all times.

Although the accuracy of the high-order model is relatively high, its structure is complex and the amount of calculation is large, making it difficult to apply largescale load adjustment capabilities. Therefore, from the point of view of simplification, the time-varying relationship between indoor temperature and electric heating power can be further connected to obtain the current first-order thermodynamic equivalent thermal parameter model. That is, this project uses the standard user model to describe the dynamic process of electric heating as:

$$
\frac{d T}{d t}=\frac{P_{\text {heat }}(t)-\left(T_{\text {in }}(t)-T_{\text {out }}(t)\right) \cdot K \cdot S}{c_{\text {air }} \cdot \rho_{\text {air }} \cdot V}
$$

Where $P_{\text {heat }}(t)$ is the building electric heating in the moment $\mathrm{t}, T_{\text {in }}(t)$ is the indoor temperature, $T_{\text {out }}(t)$ is the outdoor temperature, $K$ is the overall thermal conductivity of the external surface of the building, $S$ is the external surface area of the building, $V$ is the volume of the space inside the building, $c_{\text {air }}$ is the specific heat capacity of the air, $\rho_{\text {air }}$ is the air density of a given building.

This differential model relationship with continuous state describes the thermoelectric coupling relationship of the electric heating temperature control load, simplifies the building thermodynamic model, reveals the building transient thermal balance relationship through th4e indoor temperature change rate, and is widely applicable to those with thermal energy control capabilities Modeling of constant temperature control load and research on demand response.

\section{Thermal load adjustability model for thermal storage electric heating}

The adjustability model of thermal storage electric heating mainly includes two parts, the adjustability model of building heat load and the adjustability model of thermal storage device, which are added together to obtain the adjustability model of thermal storage electric heating. The steps to build the model will be described in detail below.

\subsection{The main influencing factors of heat load adjustability analysis}

(1) Randomness of thermodynamic parameters

The random change of parameters such as the heat capacity of the air in the building space and the comprehensive heat transfer coefficient between the interior and exterior of the building will have a certain impact on the thermal load adjustable ability.

(2) Thermal comfort temperature range

The electric heating load supplies heat by regulating the power to maintain the set indoor temperature ${ }^{[5]}$. Therefore, the size of the thermal comfort temperature is one of the important factors that affect the ability of electric heating to adjust the load. The thermal comfort temperature is usually a range value. Based on fuzzy mathematics, thermal comfort temperature can be expressed by membership function, as shown in the Fig.1.

(3) Outdoor temperature

Outdoor temperature, radiation intensity, wind speed, humidity and other external weather conditions affect the heat load demand of the building, especially the outdoor temperature change affects the indoor temperature change rate, which affects the duration of maintaining the regulated power, and then affects the heat load adjustability.

\subsection{Heat load adjustability model}

The first step is to determine the external conditions, which mainly include three aspects: building characteristics, thermal comfort temperature range, and external ambient temperature.

(1) The external surface area of the building $S$, the volume of the space inside the building $V$, the overall thermal conductivity of the external surface of the building $K$, the specific heat capacity of the air $C_{\mathrm{a}}$, and the air density of a given user $\rho_{a}$.

(2) Considering the thermal comfort temperature of residents, determine the indoor temperature adjustment range, the maximum heating power configured with the building electric heating $P_{\mathrm{sn}}$.

(3) The current indoor temperature $T_{\text {in }}$ is selected as the initial temperature $T_{0}$ when the load regulation capability is evaluated, and the outdoor temperature $T_{\text {out }}$ is selected as the ambient temperature in the time period corresponding to the weather forecast.

The second step is to determine the current heat load.

(4) Substituting the previously obtained data into the heat load model (as equation 1), the indoor temperature change rate is 0 , and the power value $P_{0}$ that maintains the current indoor temperature is determined. 


$$
P_{0}=\left(T_{\text {in }}-T_{\text {out }}\right) K \cdot S
$$

The third step is to calculate the heat load adjustable capacity.

(5) Regard the indoor temperature adjustment range $\left[T_{\text {down }}, T_{\text {up }}\right]$ as the constraint condition, $P_{0}$ as the reference value, continuously increase or decrease the power adjustment value at a certain power interval (recommended $P_{s n} / 10$ ), calculate the temperature change value according to the building electric heating heat balance. If $P_{\text {con }}>0$, corresponds to the temperature rise process, When the indoor temperature rises to $T_{\text {up }}$, the time cutoff. If $P_{\text {con }}<0$, corresponding to the temperature drop process, when the temperature drops to $T_{\text {down }}$, the time cutoff. Then the temperature can continue to be maintained in the thermal comfort zone when the power is adjusted. In the iterative process, the relationship between the indoor temperature and the time $t_{\text {con }}$ is determined at the same time, and the time that can be maintained for the given regulated power is obtained.

$$
d T=\frac{P_{0}+P_{c o n}-\left(T_{i n}-T_{o u t}\right) K \cdot S}{C_{a} \cdot \rho_{a} \bullet V} d t
$$

The fourth step is to calculate the load adjustability

$$
W_{\text {buildcontrol }}=\left[-\int_{0}^{t_{\mathrm{con} 2}} P_{\text {con2 }}(t) \mathrm{d} t, \int_{0}^{t_{\mathrm{con} 1}} P_{\text {con } 1}(t) \mathrm{d} t\right]
$$

\subsection{Thermal load adjustability model for Heat storage device and thermal storage electric heating system}

The adjustability of thermal storage electric heating is composed of the adjustability of the building heat load and the adjustability of the thermal storage device ${ }^{[1]}$. As mentioned in the previous section, the adjustability of building heat load is determined by the capacity and operating status of the heat storage device. The calculation of the adjustability model of regenerative electric heating includes 3 steps:

(1) Determine the maximum heat storage temperature in the heat storage device $T_{\text {set }}$, the water supply temperature on the primary grid side $T_{\text {high }}$, the return water temperature on the primary grid side $T_{l o w}$, the volume of the heat storage medium $V_{\text {sto }}$, the density of the heat storage medium $\sigma_{\text {sto }}$, and the specific heat capacity of the heat storage medium $C_{\text {sto }}$.

(2) adjustability of heat storage device is as follows. $W_{\text {heatstocontrol }}=\left[-\left(T_{\text {set }}-T_{\text {high }}\right) \cdot V_{\text {sto }} \bullet \rho_{\text {sto }} \cdot C_{\text {sto }},\left(T_{\text {high }}-T_{\text {low }}\right) \cdot V_{\text {sto }} \bullet \rho_{\text {sto }} \cdot C_{\text {sto }}\right]$

(3) Regulating potential of regenerative electric heating is as follows.

$$
W=W_{\text {buildcontrol }}+W_{\text {heatstocontrol }}
$$

\section{Example analysis}

This calculation example is a thermal storage electric heating project. It uses water thermal storage, power supply capacity of $1 \mathrm{MVA}$, and 120 cubic meters of hot water storage tanks. The heating construction area is 5800 square meters, including 3200 square meters for residential buildings. The basic operation mode is from 23 o'clock in the night to 6 o'clock in the next day. During the valley period, the boiler is started, while it is directly supplied to the user side, while storing heat to the water tank, the water temperature of the hot water storage tank is heated to $90{ }^{\circ} \mathrm{C}$, and the hot water storage tank is used at other times The hot water exchanges heat for heating.

The 24-hour data on a certain day in December is calculated for this paper. In the calculation of the initial heat load, the outdoor temperature uses weather forecast data, and the indoor temperature is maintained at $20^{\circ} \mathrm{C}$. The initial heat load power of the building is shown in Figure 1.

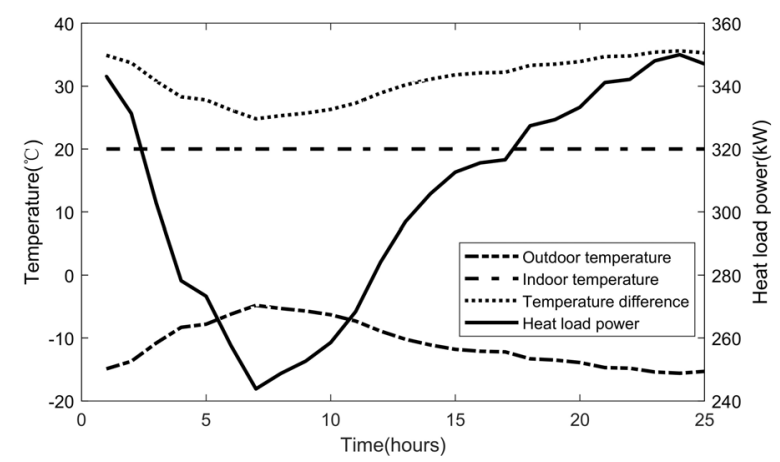

Fig.1The initial heat load power

The maximum water temperature of the heat storage device is $90^{\circ} \mathrm{C}$ and the minimum temperature is $80^{\circ} \mathrm{C}$, so the heat storage capacity of the heat storage device is $5040 \mathrm{~kJ}$. According to the operation mode of the thermal storage electric heating equipment, the heat storage heat of the thermal storage electric heating device is shown in Figure 2.

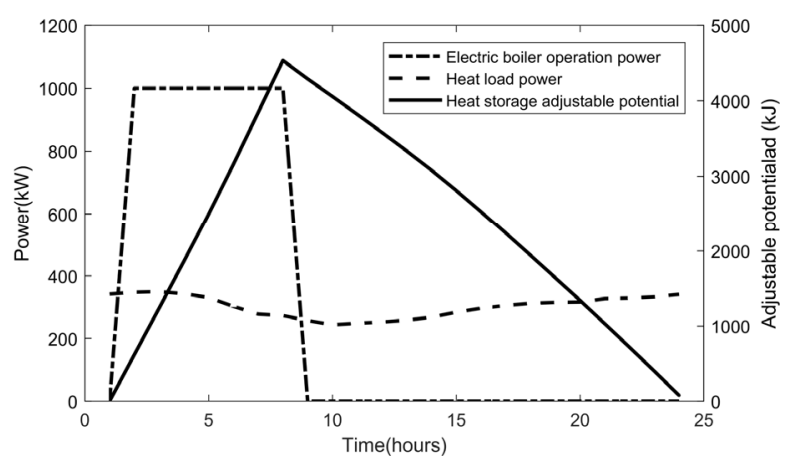

Fig.2.Thermal load adjustability model for Heat storage device

The adjustable range of building indoor temperature is set to $\left[18^{\circ} \mathrm{C}, 22^{\circ} \mathrm{C}\right]$, the height of the building is assumed to be 2.5 meters, the specific heat capacity of the air is $1.003 \mathrm{~kJ} / \mathrm{kg} /{ }^{\circ} \mathrm{C}$, and the air density is $1.205 \mathrm{~kg} / \mathrm{m} 3$. In the current regenerative electric heating and operating mode, the adjustable power and adjustable power of the thermal storage electric heating system 
considering the thermal storage capacity of the thermal storage device are shown in Figure 3.

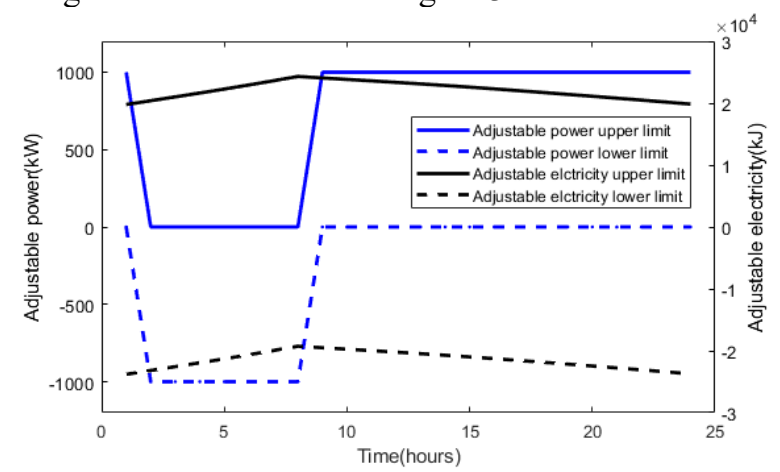

Fig.3Thermal load adjustability model for thermal storage electric heating system

\section{Conclusion}

Considering the building characteristics, outdoor temperature, indoor comfort and other factors, the building heat load adjustability model is established, and heat storage capacity of the heat storage device is established, the two are added together. An evaluation model of the adjustability of regenerative electric heating building is obtained. This model not only considers the building comfort and the adjustment potential of the building where it is located, but also considers the adjustment potential of the heat storage device, which can more comprehensively and accurately evaluate the building load adjustment potential of thermal storage electric heating. The calculation example shows that the adjustability of the building's thermal load is nearly four times that of the thermal storage device. The adjustability evaluation model of the thermal storage electric heating system proposed in this paper can more accurately assess the adjustability of the electric heating system, and provides strong support for the thermal storage electric heating system to cut peaks and fill valleys and absorb large-scale wind power.

\section{Acknowledgments}

This paper is one of the phase results of the Science and Technology Project of State Grid Corporation, "Application of Optimal Configuration and Interactive Key Technology of Thermal Storage Electric Heating" (5400-201914172A-0-0-00).

\section{References}

1. Xu Jiayi, Li Zhanxin. (2019) Energy Storage Electric Heating Technology - An Effective Supplement to Central Heating. China Resources Comprehensive Utilization,37(09):99-101.

2. Wu Bing. (2021) Discussion on the Value of Load Density of Office Buildings. Building Electricity, 40(04):8-12.

3. Zhang J, Tian Q, Wang M P. (2014) Heating Load Prediction for Heating Systems Based on Support
Vector Regression with Cross Validation. Journal of North University of China.

4. Chen Feixiang, Xu Jianqun, Wang Chenyang, Song Zhen, Liu Mingtao. (2015) Research on Building Cooling and Heating Load Prediction Model on User's Side in Energy Internet System. Proceedings of the CSEE,35(14):3678-3684.

5. Li Yaping, Yao Jianguo, Yong Taiyou, et al. (2017) Study on evaluation method of resident power and response potential for residential temperaturecontrolled load. Proceedings of the CSEE,37(19): 5519-5528. 